\title{
CAPN6 in disease: An emerging therapeutic target (Review)
}

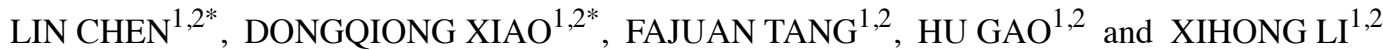 \\ ${ }^{1}$ Department of Emergency Medicine, West China Second University Hospital, Sichuan University; \\ ${ }^{2}$ Key Laboratory of Birth Defects and Related Diseases of Women and Children (Sichuan University), \\ Ministry of Education, Chengdu, Sichuan 610041, P.R. China
}

Received May 19, 2020; Accepted August 18, 2020

DOI: $10.3892 / \mathrm{ijmm} .2020 .4734$

\begin{abstract}
As a member of the calpain protein family, calpain6 (CAPN6) is highly expressed mainly in the placenta and embryos. It plays a number of important roles in cellular processes, such as the stabilization of microtubules, the maintenance of cell stability, the control of cell movement and the inhibition of apoptosis. In recent years, various studies have found that CAPN6 is one of the contributing factors associated with the tumorigenesis of uterine tumors and osteosarcoma, and that CAPN6 participates in the development of tumors by promoting cell proliferation and angiogenesis, and by inhibiting apoptosis, which is mainly regulated by the phosphatidylinositol 3 kinase $(\mathrm{PI} 3 \mathrm{~K}) /$ protein kinase B (Akt) pathway. Due to its abnormal cellular expression, CAPN6 has also been found

Correspondence to: Professor Xihong Li, Department of Emergency Medicine, West China Second University Hospital, Sichuan University, No. 20, Section 3, Renmin South Road, Chengdu, Sichuan 610041, P.R. China

E-mail: lixihonghxey@163.com

${ }^{*}$ Contributed equally
\end{abstract}

Abbreviations: CAPN6, calpain6; PI3K, phosphatidylinositol 3 kinase; AKT, protein kinase B; CysPc, calpain-like cysteine protease sequence motif defined in the conserved domain database at the National Center for Biotechnology Information (cd00044); PC, protease core; $\mathrm{PC} 1$, protease core domain 1; $\mathrm{PC} 2$, protease core domain 2; $\mathrm{C} 2$, conserved domain 2 originally defined for protein kinase $\mathrm{C}$; CBSW, calpain-type $\beta$-sandwich; PEF, penta-EF-hand (E, E-helix; F, F-helix); GEF-H1, officially known as ARHGEF2; LGMD2A, limb girdle muscular dystrophy type 2A; UtLMs, uterine leiomyomas; Rac1, Rac Family Small GTPase1; PAK1, p21-activated kinase1; VEGF, vascular endothelial growth factor; bFGF, basic fibroblast growth factor; HUVECs, human umbilical vein endothelial cells; BrdU, 5-bromodeoxyuridine; EDN-1, endothelin-1; $\mathrm{NF}-\kappa \mathrm{B}$, nuclear factor- $\kappa$-gene binding; ERK1/2, extracellular regulated protein kinase $1 / 2$; CSC, cancer stem cells; miRNA/miRs, microRNAs; HNSCC, head and neck squamous cell carcinoma; HI, hypoxia-ischemia; WMI, white matter injury; EJC, exon junction complex; LDL, low-density lipoprotein; YWHAE, tyrosine 3-monooxygenase/tryptophan 5-monooxygenase activation protein, epsilon polypeptide

Key words: calpain6, cell proliferation, apoptosis, therapeutic target to be associated with a number of diseases, such as white matter damage and muscular dystrophy. Therefore, CAPN6 may be a novel therapeutic target for these diseases. In the present review, the role of CAPN6 in disease and its possible use as a target in various therapies are discussed.

\section{Contents}

1. Overview of CAPN6

2. CAPN6 in disease: An introduction

3. CAPN6 in tumors

4. CAPN6 in neurological diseases

5. CAPN6 in vascular diseases

6. CAPN6 in muscle disorders

7. CAPN6 in skin diseases

8. Conclusions and future perspectives

\section{Overview of CAPN6}

Discovery. Calpain is a $\mathrm{Ca}^{2+}$-dependent cysteine protease (1). There have been 16 calpain genes identified to date (2). In 1997, Dear et al discovered 2 new calpain genes in vertebrates, one of these being calpain6 (CAPN6) (3), which is also known as CANPX and calpamodulin (4). CAPN6 is located on chromosome Xq23 (5). The mRNA expression of CAPN6 has been identified to be high in the placental chorionic plate, particularly during embryogenesis with a stronger expression signaling the somite, the mandibular tissue of the first branchial arch, developing skeletal muscle, the myocardium, epithelial cells bordering the fourth ventricle, bronchial epithelial cells, the tips of lung buds, the sacs of the lungs and kidney $(3,6,7)$. However, CAPN6 expression is significantly downregulated after birth (6).

Structure. Based on the differences in the C-terminal domains of members within the calpain protein family, calpain is divided into 2 groups, classic (typical) and non-classic (atypical) (8).

Classic calpains, such as CAPN1, 2, 3, 8, 9, 11, 12, 13 and 14 comprise a large subunit $(80 \mathrm{kDa})$ with catalytic activity and a small subunit $(28 \mathrm{kDa})$ with regulatory activity $(2,4,8)$. The large subunit contains 4 domains, namely I, II, III and IV. Located on the N-terminus, domain $\mathrm{I}$ is the region where autolysis occurs when the large subunit is activated by $\mathrm{Ca}^{+}$, a prerequisite for 
the hydrolysis of calpain. Domain II is the key site for hydrolytic activity with a conserved calpain-like cysteine protease sequence motif defined in the conserved domain database at the National Center for Biotechnology Information (cd00044; $\mathrm{CysPc}$ ) catalytic region, which consists of 2 protease core (PC) domains (PC1 and PC2). Related to the conserved domain 2 originally defined for protein kinase $\mathrm{C}(\mathrm{C} 2)$, domain III is the calpain-type $\beta$-sandwich (CBSW) domain that plays a regulatory role by undergoing conformational changes during calcium-binding. Domain IV at the C-terminus with a penta-EF-hand $(\mathrm{PEF})$ is the calcium-binding site $(2,9,10)$. Moreover, the small subunit contains 2 domains, namely $\mathrm{V}$ and VI. The glycine-rich domain $\mathrm{V}$ is a hydrophobic region where calpain binds to cell membranes or membrane proteins. Similar to domain IV, the PEF-containing domain VI is the calmodulin binding region (11).

Non-classic calpains, such as CAPN5, 6, 7, 10, 13, 15 and 16 have a different motif that replaces the IV domain (4). Although most residues of CAPN6 are highly conserved among members of the calpain family, it lacks a C-terminal calmodulin-like domain, and it contains a CBSW domain instead of a PEF domain at the catalytic subunit $(7,12)$. Due to the lack of a key active site that contains catalytic cysteine residues for proteolytic activity, it is believed that the CAPN6 protein may employ different sites for proteolysis. Since the entire coding region exhibits a significant homology with TRA-3 that is involved in the sex determination of nematodes, the C-terminal structure of CAPN6 is defined as a forked C2 domain (also known as domain T) (3,5,13-15), rather than domain IV (summarized in Fig. 1).

Physiological function. Classic calpain is a highly conserved protease widely expressed in a number of tissues that participates in various physiological and pathological processes of the cell, including cell proliferation, apoptosis, cell migration and invasion, cytoskeleton remodeling, signal transduction, etc. $(4,16-18)$.

To date, there are only a few studies available that address the physiological functions of CAPN6 protein, even though CAPN6 has a unique structure which may confer a different functional and regulatory mechanism from that of members of the classic calpain $(7,12,19-21)$. During cytokinesis, CAPN6 is co-localized to the microtubule structure, including the central spindle and intermediates. As a microtubule-stabilizing protein, the domain III of CAPN6 binds to microtubules to induce stabilization through a non-proteolytic activity (7). Conversely, the inhibition of CAPN6 destroys the cytoskeletal tissues and the absorption capacity of cells, which then lowers the microtubule stability, the levels of acetylation and b3-integrin subunit proteins in osteoclasts, further damages the factors that regulate osteoclast cytoskeleton function, and ultimately causes cytoskeleton remodeling (19).

Rac Family Small GTPase1 (Rac1GTPase) and vimentin are the key factors for cell movement (22). CAPN6 inhibits the activity of Rac1GTPase through the interaction with Rho guanine nucleotide exchange factor GEF-H1 (officially known as ARHGEF2) in order to control lamellipodial formation and cell movement (20). The crosstalk between microtubules and actin as the main cytoskeletal components is essential for cell migration, diffusion and cytokinesis. Changes in microtubule stability have an impact on microtubule-actin interactions and cell movement (23). Therefore, the inactivation of CAPN6 not only causes the instability of microtubules, the loss of acetylated $\beta$-tubulin, and the reorganization of actin, it also leads to the formation of lamellipodium, laminar pseudofoot and laminar folds, which affect the actin tissues, and ultimately promotes cell movement and diffusion $(7,20)$.

The activity of microtubule cytoskeleton and small GTPases are the key regulators of muscle cell differentiation and skeletal muscle development $(24,25)$. CAPN6 regulates microtubule dynamics and actin reorganization by affecting the activity of Rac1GTPase. CAPN6 is highly expressed in embryonic muscle. The knockout of CAPN6 gene in mice can promote the differentiation of the muscle cells into myotubes. CAPN6 mRNA and CAPN6 protein can be detected in the regenerated skeletal muscle of adult mice. Compared with that of $C A P N 6^{\text {lacZ/+ }}$ mice, the regenerated skeletal muscle of $C A P N 6^{\text {lacZ/lacZ }}$ mice has more nuclei per muscle fiber and a larger cross-sectional area (21). Therefore, CAPN6 deficiency can promote skeletal muscle differentiation during skeletal muscle development and regeneration.

In addition, studies have demonstrated that in uterine leiomyoma, cervical cancer, osteosarcoma and liver cancer cells, an increased level of CAPN6 expression can promote cancer cell proliferation and inhibit cell apoptosis $(12,26-28)$. In white matter damage, a decreased level of CAPN6 expression can promote oligodendrocyte precursor cell apoptosis (29).

Overall, CAPN6 plays a role in maintaining cell stability, in controlling cell movement and diffusion, and in inhibiting skeletal muscle differentiation and growth. CAPN6 also plays an anti-apoptotic role.

\section{CAPN6 in disease: An introduction}

Due to the abnormal protein expression levels, members of the calpain protein family have been found to be associated with the development of a number of diseases, such as Alzheimer's disease, gastric cancer, muscular dystrophy, diabetes, atherosclerosis, psoriasis, etc. (30-35). These are common diseases with a high incidence rate and a significant impact on society. Based on the different mechanisms involved in the pathogenesis of diseases, calpain-related diseases can be classified into 3 categories as follows: Diseases caused or aggravated by calpain activity, diseases caused by pathogenic microorganisms which use the host and/or their own calpain, and infection and survival caused by calpain gene defects (36). Calpain has been demonstrated as an effective therapeutic target for a number of diseases (37). Treatment strategies include the inhibition of calpain, such as $\alpha$-ketoamide inhibitor A-705253 (also known as BSF 419961 and CAL 9961) in treating Alzheimer's disease; and the activation of calpain (direct or replacement), such as the restoration or compensation of the loss of CAPN3 function in improving limb-girdle muscular dystrophy type 2A (LGMD2A) $(36,38,39)$. As a target of disease treatment, the use of calpain is very challenging as classic calpains are expressed in almost all cells. However, non-classic calpains are mainly expressed in specific tissues and organs, exhibiting their unique potential. In recent years, with an improved knowledge of physiological functions of CAPN6, a number of diseases have been identified to be 

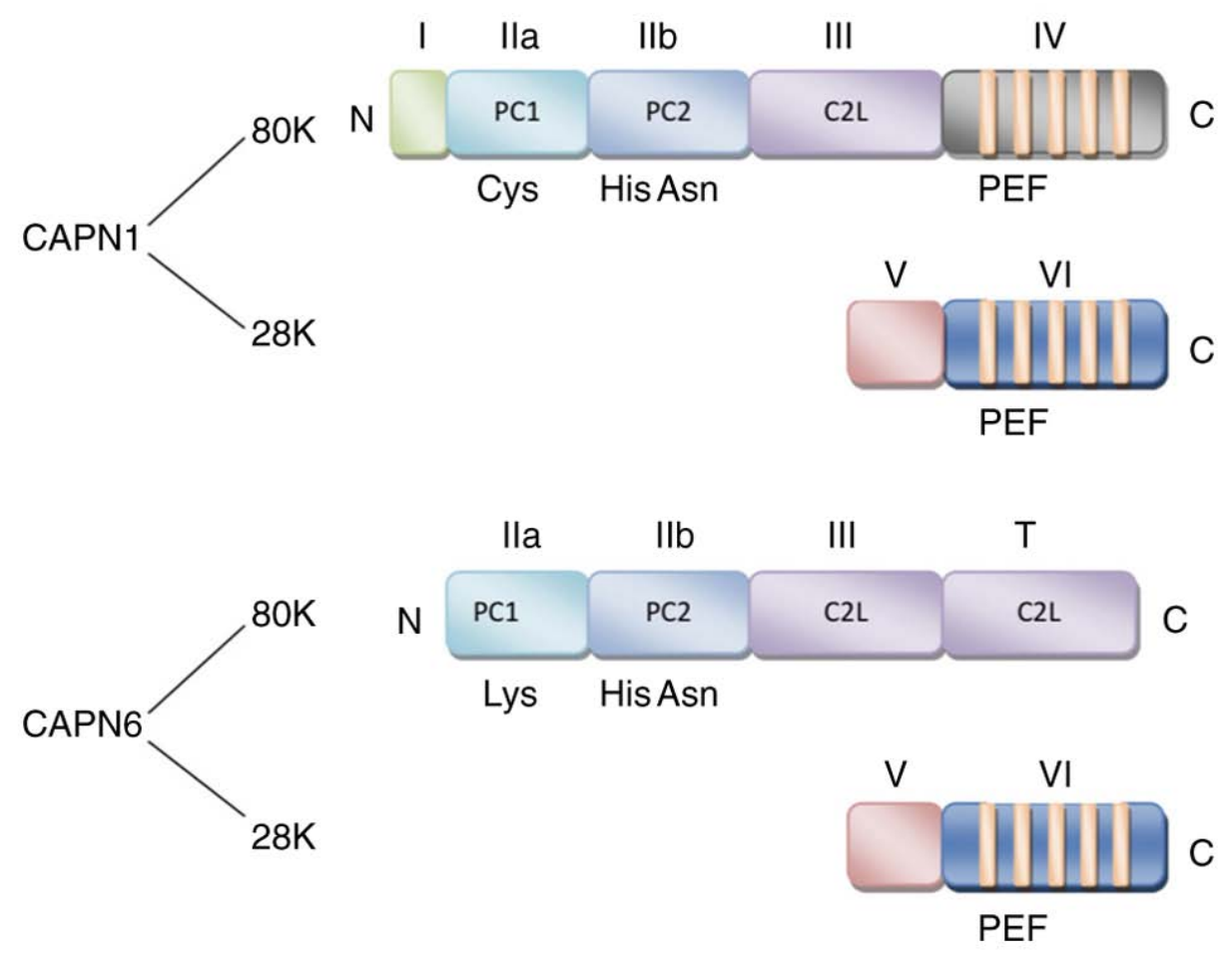

Figure 1. Domain structure of CAPN1 and CAPN6. CAPN1 comprise a large subunit (80 kDa) and a small subunit (28 kDa). The large subunit contains four domains, namely I, II, III and IV, domain IV at the C-terminus with a PEF. The small subunit contains two domains, namely V and VI. The main difference in the structure of CAPN6 is that it has a domain T at the C-terminus, which contains a CBSW domain instead of a PEF domain at the catalytic subunit. CAPN calpain; PEF, penta-EF-hand (E: E-helix; F: F-helix); CBSW, calpain-type $\beta$-sandwich.

specifically linked to CAPN6 (Table I), including tumors, neurological diseases, vascular diseases, muscle diseases and skin diseases $(21,28,40-47)$. Among all the CAPN6-associated diseases, tumors have gained the most research attention.

\section{CAPN6 in tumors}

Uterine tumors. Uterine leiomyomas (UtLMs) are the most common benign tumor of the female reproductive system. Overexpressed CAPN6 in UtLMs interacts with other abnormally expressed factors, such as neuron navigator 2 (NAV2), kinesin family member 5C (KIF5C), doublecortin (DCX), etc., which may lead to the pathological transformation of the normal myometrium through related biochemical pathways $(26,48)$. It has recently been found that, whilst CAPN6 may regulate proliferation and apoptosis in UtLM through the Rac1/p21-activated kinase 1 (PAK1) signaling pathway, the downregulation of CAPN6 inhibits cell proliferation and promotes cell apoptosis in UtLM (49). Therefore, the overexpression of CAPN6 promotes the occurrence and development of UtLM.

CAPN6 has been found to be overexpressed in the tissues of other malignant tumors of the uterus, such as leiomyosarcoma, endometrial stromal sarcoma and cervical cancer $(12,40,50-52)$. As previously demonstrated, there is a modest association between the intensity of CAPN6 expression and tumor subtype, but not tumor stage or survival rate (50).

The expression of CAPN6 in cervical cancer is regulated by the phosphatidylinositol 3 kinase (PI3K)/protein kinase B (Akt) pathway (12), which plays an important role in a number of cellular processes, and promotes tumor growth and survival (53). The PI3K/Akt pathway inhibits the proteasome degradation of CAPN6 protein through glycogen synthase kinase (GSK)-3 $\beta$, increases the stability of CAPN6 protein, upregulates CAPN6 mRNA expression, and uses transcription factors, such as activator protein 1 (AP1), forkhead box D3 (FoxD3) and organic cation transporter 1 (Oct-1) to stimulate the activity of the CAPN6 promoter (12). A main prerequisite for tumor formation and progression is to prevent apoptosis, and promote cell proliferation and angiogenesis. Cisplatin is used to induce cervical cancer cell apoptosis by activating the caspase-3 pathway (54). A previous study using cervical cancer cells (HeLa cells) transfected with CAPN6 cDNA (Calpain 6) or CAPN6 siRNA (siCalpain 6) demonstrated that CAPN6 antagonized cisplatin-mediated apoptosis by inhibiting caspase-3 activity (51). Consistently, another CAPN6 knockdown study using HeLa cells demonstrated an upregulation of caspase-3 and a downregulation of Bcl-2 (12), indicating that CAPN6 induced resistance to apoptosis. HeLa cells with a downregulated CAPN6 expression grow at a more rapid rate than their parental cells, with a reduced $\mathrm{S}$ phase and cell-specific cyclin (cyclin D1) expression, suggesting that CAPN6 can promote the proliferation of cancer cells (12). Vascular endothelial growth factor (VEGF) and basic fibroblast growth factor (bFGF) promote endothelial cell migration, proliferation and angiogenesis, and activate calcium signal regulation in endothelial cells (55-57). Since the secretion of VEGF and bFGF is related to cervical cancer (58), a study using human umbilical vein endothelial cells (HUVECs) demonstrated that CAPN6 overexpression enhanced the VEGF-induced invasiveness of endothelial cells, and the tissue formation of endothelial cell network, indicating that calpain 6 
Table I. CAPN6 expression and active pathways in tissues in different diseases.

\begin{tabular}{|c|c|c|c|}
\hline Disease & Effect & Active pathway ${ }^{\mathrm{a}}$ & Refs. \\
\hline \multicolumn{4}{|l|}{ Tumors } \\
\hline \multicolumn{4}{|l|}{ Uterine tumors } \\
\hline Uterine leiomyomas & Overexpression & Rac1/PAK1/CAPN6 & $(26,49)$ \\
\hline Uterine sarcoma & Overexpression & - & $(40,50,52)$ \\
\hline Cervical cancer & Overexpression & PI3K/AKT/CAPN6 & $(12,51)$ \\
\hline Osteosarcoma & Overexpression & EDN-1/ERK1/2, PI3K/AKT, NF-кB/CAPN6 & $(27,41,60,61)$ \\
\hline Liver cancer & Overexpression & PI3K/AKT/CAPN6 & $(12,28,70)$ \\
\hline Head and neck squamous cell carcinoma & Low expression & - & $(42)$ \\
\hline \multicolumn{4}{|l|}{ Neurological diseases } \\
\hline White matter injury & Low expression & miR-142-3p/miR-466b-5p/CAPN6 & $(29)$ \\
\hline Prion diseases & Overexpression & - & $(43)$ \\
\hline \multicolumn{4}{|l|}{ Vascular diseases } \\
\hline Atherosclerosis & Overexpression & CWC22/EJC/Rac1 & $(46,78-80)$ \\
\hline Target organ damage in hypertension & Low expression & - & (44) \\
\hline Type 2 diabetic nephropathy & Overexpression & - & $(45)$ \\
\hline \multicolumn{4}{|l|}{ Muscular diseases } \\
\hline Muscular dystrophy & Overexpression & - & $(21)$ \\
\hline Muscular atrophy & Overexpression & - & $(21)$ \\
\hline \multicolumn{4}{|l|}{ Skin diseases } \\
\hline Atopic dermatitis & - & Key factors related to YWHAE & $(47)$ \\
\hline
\end{tabular}

promotes HUVEC invasion, migration and angiogenesis. Since calpain 6 is a $\mathrm{Ca}^{2+}$-dependent cysteine protease, it is considered that the intracellular calcium signals induced by VEGF or bFGF can possibly activate CAPN6 (51). A recent study found that the binding between the domain III of CAPN6 and the C-terminus of VEGFA, produced a unique interaction that leads to the secretion of VEGF, which in turn increases and promotes angiogenesis (59). Although it is generally accepted that CAPN6 plays a role in promoting the occurrence and development of cervical cancer, CAPN6 is not associated with cancer cell migration and epithelial-mesenchymal transition (12).

Osteosarcoma. Osteosarcoma is one of the most common primary malignant bone tumors. Metastasis and recurrence are important factors leading to a poor prognosis. Chemoresistance is the main cause of the poor treatment efficacy and disease recurrence in patients with osteosarcoma.

It has been demonstrated that CAPN6 is expressed in human osteosarcoma tissue, metastatic bone and lung, and the majority of recurrent tumors at high levels (41). The CAPN6 level in the primary tumor is found to be negatively associated with the chemotherapeutic response. Compared with original U2OS sensitive cells, cells with a resistant U2OS osteosarcoma origin have been shown to express higher CAPN6 levels (27).

The inhibition of CAPN6 expression in osteosarcoma cells can reduce the incorporation of 5-bromodeoxyuridine (BrdU) into DNA, reduce cell proliferation, increase spontaneous apoptosis, rescue the apoptotic response of resistant cells, and increase the sensitivity to cytotoxic drugs. Therefore, CAPN6 promotes osteosarcoma cell proliferation, survival and growth, and provides protection by increasing the chemical resistance of cells (27). CAPN6 is a downstream molecule of endothelin-1 (EDN-1) signaling during embryonic development (7). EDN-1 can induce the continuous activation of the nuclear factor- $\kappa$-gene binding $(\mathrm{NF}-\kappa \mathrm{B})$, extracellular regulated protein kinase $1 / 2($ ERK1/2) and AKT pathways, and can promote the expression of CAPN6 in osteosarcoma $(60,61)$. Tumor cytochemical resistance is associated with the activation of the NF- $\mathrm{B}$, Ras/MAPK and PI3K/AKT pathways $(62,63)$, further demonstrating the association between CAPN6 expression and osteosarcoma cell chemical resistance. In addition, in liposarcoma, it has been found that the higher the tumor grade, the higher the CAPN6 expression (64), indicating that the expression of CAPN6 is associated with the malignancy of sarcoma.

Syndecan-2 is a key regulator of cell death, and has the function of promoting apoptosis. Since there is a decreased level of expressed syndecan-2 in osteosarcoma tissue, it is inferred that the abnormal expression or induction of syndecan-2 in osteosarcoma is associated with the imbalanced apoptosis of tumor cells (65). The overexpression of syndecan-2 in osteosarcoma cells can enhance the apoptotic response to cytotoxic drugs, restore the sensitivity of drug-resistant osteosarcoma cells to chemotherapeutic drugs, decrease the levels of CAPN6 in the tumor cells and ultimately inhibit tumor 


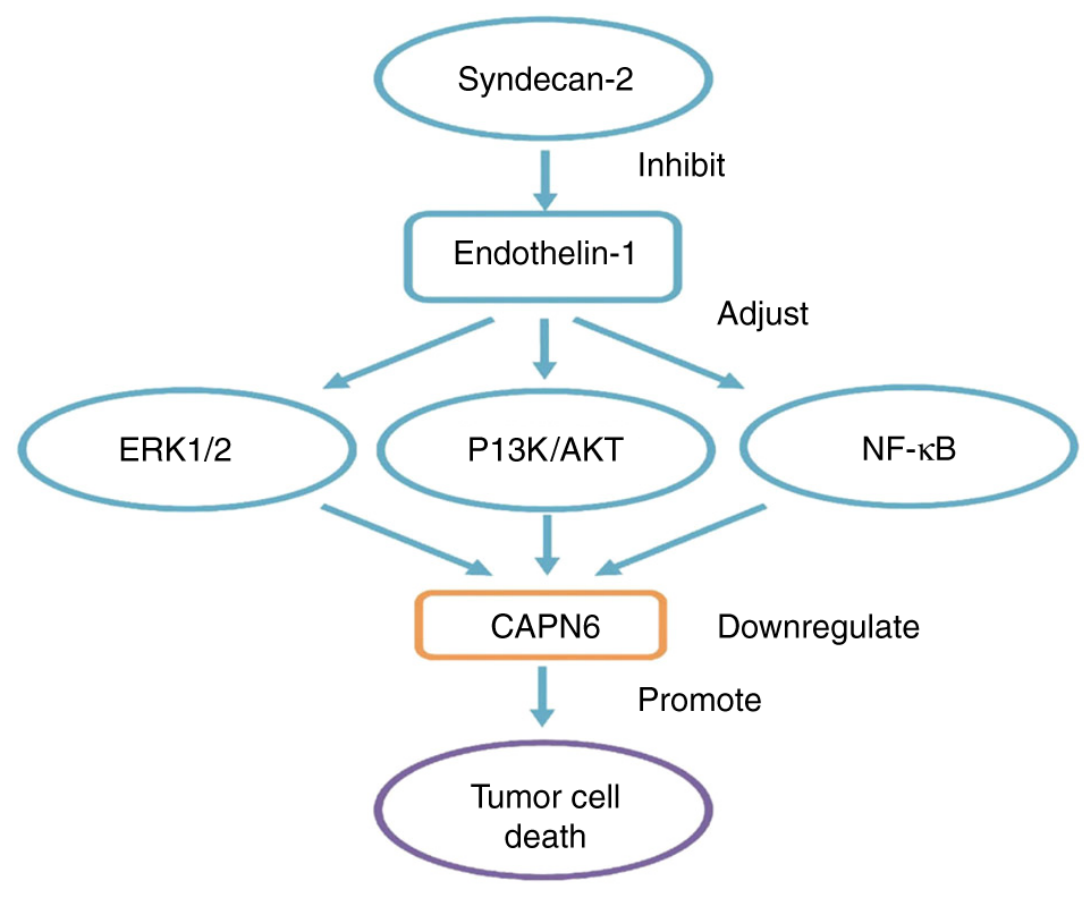

Figure 2. In osteosarcoma, CAPN6 involves related signal pathways. CAPN6 is a downstream molecule of EDN-1 signaling. EDN-1 can induce the continuous activation of NF- $\mathrm{KB}$, ERK1/2 and AKT pathways, and promote the expression of CAPN6 in osteosarcoma. Syndecan-2 is a key regulator of cell death. Overexpression of syndecan-2 in osteosarcoma cells can decrease the levels of CAPN6 in the tumor cells, and can alter EDN-1 signaling. PI3K/AKT and $\mathrm{NF}-\mathrm{\kappa B}$ are new pathways controlled during their pro-apoptotic process. In cells with syndecan-2 overexpression, steady-state activation of ERK1/2 and AKT is low. Therefore, syndecan-2 can inhibit EDN-1 signal transduction, and down-regulate CAPN6 expression through ERK1/2, PI3K/AKT and NFkB pathways

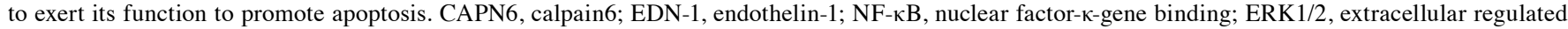
protein kinase $1 / 2$; AKT, protein kinase B.

growth; thus, a decrease in CAPN6 expression contributes to syndecan-2-induced apoptosis $(41,66)$. EDN-1 is overexpressed in osteosarcoma to promote the invasion, metastasis and survival of bone cancer cells, and to resist cisplatin-induced apoptosis through $\mathrm{EDN}_{\mathrm{A}}$ receptor (67). Syndecan-2 can alter EDN-1 signaling. The PI3K/AKT and NF- $\kappa \mathrm{B}$ pathways are controlled during the pro-apoptotic process. In cells with syndecan-2 overexpression, the steady-state activation of ERK1/2 and AKT is low $(60,61)$. Therefore, syndecan-2 can inhibit EDN-1 signal transduction, and can downregulate CAPN6 expression through the ERK1/2, PI3K/AKT and $\mathrm{NF}-\kappa \mathrm{B}$ pathways to exert its function to promote apoptosis (summarized in Fig. 2).

Cancer stem cells (CSC) have been shown to promote the development of malignancies. Oct4, Nanog and Sox 2 are stem cell transcription factors that can be upregulated by hypoxia in osteosarcoma. Hypoxia can also activate NF- $\kappa \mathrm{B}$ and increase the expression of EDN-1 receptors, thereby promoting the EDN-1/NF- $\kappa$ B pathway to induce increased mRNA and protein levels of CAPN6, resulting in a marked decrease in the silencing of Oct4, Nanog or Sox 2 (68). In a previous study, in an osteosarcoma mouse model, it was found that cells expressing CAPN6 had unique tumor initiation and metastasis capabilities; CAPN6 knockdown inhibited hypoxia, promoted autophagy, prevented aging, induced cancer stem cell population depletion and blocked mouse tumor development (64). CAPN6 expression also recognizes CSCs. The metastatic potential of bone tumors is dependent on CSCs that express CAPN6, and CSCs communicate with other tumor cells through exosomes to regulate the cell migratory capacity (69).
Liver cancer. Consistent with that in cervical cancer, the enhanced expression of CAPN6 in liver cancer (70), which is regulated by the PI3K/AKT signaling pathway, plays a role in promoting cancer cell proliferation and inhibiting apoptosis, but not necessarily tumor metastasis (12). MicroRNAs (miRNAs or miRs) play a key role in post-transcriptional gene regulation (71), and an miRNA imbalance may lead to the inactivation of liver cancer suppressor genes and the activation of oncogenes, mainly by inhibiting the expression of its target genes (72). miR-449a expression is decreased in liver cancer (27). In a previous study, luciferase assay confirmed that CAPN6 and POU2F1 are the target genes of miR-449a (29). The downregulation of CAPN6 or POU2F1 increases the levels of Bax, decreases the levels of $\mathrm{Bcl}-2$, promotes $\mathrm{G} 1$ phase arrest, and inhibits cancer cell proliferation and induces apoptosis, thereby preventing the occurrence and development of liver cancer (28).

Head and neck squamous cell carcinoma (HNSCC). Xiang et al examined CAPN6 expression in HNSCC, including tongue cancer, laryngeal cancer and nasal cancer (42). The results of their study demonstrated that the expression of CAPN6 in HNSCC tissues was significantly decreased, which was in complete contrast to the observations of other tumor studies, as mentioned above. CAPN6 expression also varies between different tumor stages of HNSCC, and it differs significantly between different $\mathrm{T}$ stages, although there is no significant difference between different $\mathrm{N}$ stages and different tumor grades. In addition, the expression of CAPN6 and the survival rate of patients with HNSCC exhibit a positive association (42). However, it is not clear whether the expression 
of CAPN6 is directly linked to the occurrence of HNSCC, or whether HNSCC tumor growth or metastasis leads to CAPN6 downregulation.

These studies have provided new knowledge for the further understanding of the pathogenesis of these tumors. CAPN6 may be used as a biomarker, and in the diagnosis, prevention and management of these tumors. Most importantly, CAPN6 may be an ideal specific therapeutic target. For uterine tumors, osteosarcoma and liver cancer that can be caused or aggravated by CAPN6 activity, the inhibition of CAPN6 may be an effective treatment strategy, particularly for chemoresistant and recurring tumors. Therefore, CAPN6 can be inhibited to produce targeted drugs (including targeted cell drugs and targeted vascular drugs) or tumor stem cells to block the initiation, proliferation or metastasis of tumor cells, to promote the occurrence of apoptosis or autophagy, and to achieve the purpose of treatment. The combination of CAPN6 targeting with anticancer drug cisplatin may also produce a synergistic effect. However, it is worth noting that studies have found the pros and cons of calpain inhibition, depending on the stage of tumor progression (73), and that CAPN6 inhibition has an opposite effect in HNSCC. Therefore, further systematic studies are warranted to determine whether CAPN6 exerts differential effects in different stages of the same tumor, and different cell types of tumors, as well as to determine whether CAPN6 targeting is beneficial for the treatment of tumors.

\section{CAPN6 in neurological diseases}

White matter injury (WMI). Studies have found that hypoxia-ischemia (HI) can lead to reduced myelin sheath, the death of oligodendrocyte precursor cells and the dysplasia of oligodendrocytes, and can alter the expression of specific mature miRNAs in demyelinating and oligodendrocyte precursor cells, ultimately resulting in WMI $(29,71,74)$. The co-expression network of miRNAs/mRNAs indicates that miR-142-3p, miR-466b-5p and miR-146a-5p have differentially expressed targets. CAPN6 is the target gene of miR-142-3p and miR-466b-5p. The abundance of CAPN6 mRNAs in $\mathrm{HI}$ is significantly decreased. Therefore, it is considered that miR-142-3p and miR-466b-5p may promote the apoptosis of oligodendrocyte precursor cells by inhibiting CAPN6, and that miR-142-3p/miR-466b-5p/CAPN6 pathway may be involved in the pathogenesis of WMI (29).

Prion diseases. Prion diseases are a group of lethal neurodegenerative disorders; however, the molecular mechanisms responsible for these diseases are poorly understood. Other members of the calpain family are associated with prion diseases and several other neurodegenerative diseases (75). Calpain-mediated proteolytic cleavage by prion protein $\left(\mathrm{PrP}^{\mathrm{Sc}}\right)$ may be an important event in prion reproduction (76). CAPN6 is overexpressed in the medulla oblongata, spinal cord, cerebellar cortex and several other areas of prion disease-affected brain tissues. CAPN6 is involved in the pathogenesis of prion diseases (43).

CAPN6 participates in different pathogenesis of WMI and prion diseases; thus, the corresponding targeted treatment strategies vary. The decreased activity of CAPN6 in WMI the promotes the development of disease. Hence, treatment strategies should consider activating or replacing CAPN6, as no calpain activator has been developed to date, at least to the best of our knowledge. The only treatment option available is calpain replacement or gene therapy (36). However, to the best of our knowledge, to date, there is no research available on the overexpression of CAPN6 in the treatment of diseases in live animals; thus, it is difficult to assess whether gene therapy will bring other effects. In prion diseases, it is speculated that prion proteins can reproduce and survive to promote disease by mediating CAPN6. Therefore, inhibiting CAPN6 to ameliorate the invasion and growth of the infecting virus may be a novel treatment strategy. For the inhibition of calpain in the treatment of neurodegenerative diseases, mature drugs are already available, such as the aforementioned $\alpha$-ketoamide inhibitor, A-705253. There are also drugs that have been tested on animals, such as Gabadul for the treatment of Parkinson's disease and Lewy body dementia $(36,77)$. Hence, therapeutic strategies with the inhibition of CAPN6 may be worthy of investigation.

\section{CAPN6 in vascular diseases}

Degenerative vascular diseases. In degenerative vascular diseases, the dysfunction of the calpain system has a prominent effect, since the increased activity of calpain can induce diseases (78). As a non-proteolytic calpain, CAPN6 disrupts the CWC22/exon junction complex (EJC) system in macrophages to affect CWC22/EJC/Racl signal transduction, and enhances the cell phagocytosis of natural low-density lipoprotein (LDL), causing the cytosolic DL cholesterol deposition in cells, thereby attenuating the clearance of dead cell corpses, and eventually promoting the progression of degenerative vascular diseases, such as atherosclerosis (46,78-80).

Hypertensive heart disease. Damage to target organs is the most harmful effect caused by hypertension. Experiments have established a model of target organ damage in hypertension. It has been found that in the heart tissue of Dahl salt-sensitive rats with high salt $(4 \% \mathrm{NaCl})$, CAPN6 and CAPN9 are downregulated by $>50 \%$, and that endogenous calpain inhibitor calpastatin is upregulated by $225 \%$. However, the specific role of CAPN6 in hypertension target organ damage is unknown, as CAPN6 lacks key amino acids in the catalytic triad and may not have proteolytic activity (44).

Diabetic nephropathy. Diabetic nephropathy is one of the comorbidities of diabetic systemic microangiopathies. Due to the higher expression levels, CAPN6 is one of the 50 functional genes that may be responsible for the occurrence of type 2 diabetes (45).

In vascular diseases, the expression and site of action of CAPN6 differ due to the nature of the disease and the different organs involved. In degenerative vascular diseases, CAPN6 mainly affects lipid metabolism by participating in the interference of mRNA splicing in macrophages. It has been found that the transgenic overexpression of calpastatin and calpain inhibitors (such as MDL28170 and BDA 410), are effective in the treatment of degenerative disorders (36). Although there is no drug that inhibits calpain-6, the development of atypical calpain inhibitors is promising. In acute cardiovascular diseases, calpain-mediated myocardial proteolysis 
is involved in ischemia-reperfusion and pressure overload mechanisms that lead to the pathogenesis. Studies performing animal experiments have indicated that calpain inhibitors can improve the symptoms of these diseases $(81,82)$. However, it has also been reported that the inhibition of calpain may lead to a decrease in endogenous calpain activity, which is unfavorable (83). This is consistent with the decrease in CAPN6 activity and the upregulation of endogenous calpain inhibitors in the hypertensive heart disease model. The inhibition of calpain can also cause cardiac dysfunction under pressure overload (82). Therefore, calpain may play multiple roles in cardiovascular diseases, either in a therapeutically inhibited or activated manner. As regards diabetic nephropathy, only the specific biological pathways and sites of action of CAPN6 involved in the pathogenesis are clarified, the treatment measures need to be further addressed.

\section{CAPN6 in muscle disorders}

Muscular dystrophy and muscle atrophy. With CAPN6 being overexpressed, the developing skeletal muscle is in a continuous cycle of degeneration and regeneration. CAPN1 is also related to muscle differentiation. CAPN1 overexpression inhibits muscle cell differentiation (84). During muscle degeneration, the expression of CAPN1 decreases, which then recovers during muscle regeneration. However, CAPN6 deficiency delays the expression of CAPN1 in regenerating skeletal muscles (21). The exact association between CAPN1 and CAPN6 needs to be clarified.

Muscular dystrophy is a muscle degeneration disease caused by genetic mutations in slow progressive symmetrical muscle weakness and atrophy; while muscle atrophy is muscle reduction and rhabdomyolysis caused by the thinning or even disappearance of muscle fibers. Studies have found that CAPN6 is one of the genes associated with LGMD2A, and its expression is up-regulated in the skeletal muscles of patients (85). As an inhibitor of skeletal muscle differentiation, CAPN6 downregulation assists the growth of skeletal muscles and the induction of pluripotent stem cells to produce skeletal muscles in vitro. Therefore, the modification of specific antibodies or siRNA to offset CAPN6 may regulate the quality of skeletal muscles, and may improve the conditions of muscular dystrophy or muscle atrophy. However, the inhibition of calpain does not necessarily improve muscle function. The inhibition of calpain through inhibitors or calpastatin overexpression can lead to the compensatory upregulation of calpain (86). Therefore, the results of such treatments need to be analyzed dialectically. Limb-girdle muscular dystrophy type 2 (LGMD2A) is a recessive genetic disease caused by CAPN3 mutation and loss of function (85). The inhibition of CAPN6 alone may not be able to treat LGMD2A completely. A combination of CAPN6 inhibition with other treatment methods may be more beneficial.

\section{CAPN6 in skin diseases}

Atopic dermatitis. Atopic dermatitis is a familial hereditary skin disease. The tyrosine 3-monooxygenase/tryptophan 5-monooxygenase activation protein, epsilon polypeptide (YWHAE) isoform located in the human keratinocytes is involved in the pathogenesis of atopic dermatitis (87). CAPN6 is one of the key factors associated with YWHAE (47). Addressing the causal association and signal transduction pathways between CAPN6 and YWHAE may facilitate the discovery of novel clinical treatments for YWHAE-related atopic dermatitis.

\section{Conclusions and future perspectives}

Calpain-related diseases are a threat to human health. The development of therapeutic drugs is the focus of current research. Although some studies have facilitated the understanding of the disease pathogenesis and the application of calpain in disease treatment, there are several aspects that require further investigations. For instance, knowledge about calpains, particularly non-classical calpains with natural advantages warrant further attention. CAPN6 has great potential as an emerging therapeutic target, although there are a number of research areas that require further clarifications, including the characteristics of CAPN6 itself, the molecular pathways involved in the associated diseases, the identification of targets, the development of CAPN6 inhibitors or activators, and the effective testing of these therapies. However, the future research direction of CAPN6 holds promise.

\section{Acknowledgements}

Not applicable.

\section{Funding}

The present study was supported by the National Key R\&D Programme of China (grant nos. 2017YFA 0104201 and 2017YFA 0104200), the National Science Foundation of China (grant nos. 81330016, 81630038 and 81771634), and the Science and Technology Bureau of Chengdu City (grant no. 2015-HM01-00424-SF).

\section{Availability of data and materials}

Not applicable.

\section{Authors' contributions}

LC and DX were involved in the conceptualization of the study. LC, DX, FT and XL were involved in software applications. LC, DX, HG and XL provided the study resources. FT, $\mathrm{HG}$ and XL also played a role in the conceptualization of the study. LC and DX were involved in the writing and preparation of the original draft, and in the writing, reviewing and editing of the study. HG and XL were involved in the processing of the figures. XL supervised the study. All authors read and approved the final manuscript.

\section{Ethics approval and consent to participate}

Not applicable.

\section{Patient consent for publication}

Not applicable. 


\section{Competing interests}

The authors declare that they have no competing interests.

\section{References}

1. Sorimachi H, Hata S and Ono Y: Calpain chronicle-an enzyme family under multidisciplinary characterization. Proc Jpn Acad Ser B Phys Biol Sci 87: 287-327, 2011.

2. Moretti D, Del Bello B, Allavena G and Maellaro E: Calpains and cancer: Friends or enemies? Arch Biochem Biophys 564: 26-36, 2014.

3. Dear N, Matena K, Vingron M and Boehm T: A new subfamily of vertebrate calpains lacking a calmodulin-like domain Implications for calpain regulation and evolution. Genomics 45 : $175-184,1997$

4. Sorimachi H, Hata S and Ono Y: Impact of genetic insights into calpain biology. J Biochem 150: 23-37, 2011.

5. Matena K, Boehm T and Dear N: Genomic organization of mouse Capn5 and Capn6 genes confirms that they are a distinct calpain subfamily. Genomics 48: 117-120, 1998.

6. Dear TN and Boehm T: Diverse mRNA expression patterns of the mouse calpain genes Capn5, Capn6 and Capn11 during development. Mech Dev 89: 201-209, 1999.

7. Tonami K, Kurihara Y, Aburatani H, Uchijima Y, Asano T and Kurihara H: Calpain 6 is involved in microtubule stabilization and cytoskeletal organization. Mol Cell Biol 27: 2548-2561, 2007.

8. Hanna RA, Campbell RL and Davies PL: Calcium-bound structure of calpain and its mechanism of inhibition by calpastatin Nature 456: 409-412, 2008

9. Glading A, Lauffenburger DA and Wells A: Cutting to the chase: Calpain proteases in cell motility. Trends Cell Biol 12: 46-54, 2002.

10. Suzuki K and Sorimachi H: A novel aspect of calpain activation. FEBS Lett 433: 1-4, 1998.

11. Campbell RL and Davies PL: Structure-function relationships in calpains. Biochem J 447: 335-351, 2012.

12. Liu Y, Mei C, Sun L, Li X, Liu M, Wang L, Li Z, Yin P, Zhao C, Shi Y, et al: The PI3K-Akt pathway regulates calpain 6 expression, proliferation, and apoptosis. Cell Signal 23: 827-836, 2011.

13. Barnes TM and Hodgkin J: The tra-3 sex determination gene of Caenorhabditis elegans encodes a member of the calpain regulatory protease family. EMBO J 15: 4477-4484, 1996.

14. Goll DE, Thompson VF, Li H, Wei W and Cong J: The calpain system. Physiol Rev 83: 731-801, 2003.

15. Mugita N, Kimura Y, Ogawa $M$, Saya $H$ and Nakao $M$ Identification of a novel, tissue-specific calpain htra-3; a human homologue of the Caenorhabditis elegans sex determination gene. Biochem Biophys Res Commun 239: 845-850, 1997.

16. Hosseini M, Najmabadi $\mathrm{H}$ and Kahrizi K: Calpains: Diverse functions but enigmatic. Arch Iran Med 21: 170-179, 2018.

17. Lebart MC and Benyamin Y: Calpain involvement in the remodeling of cytoskeletal anchorage complexes. FEBS J 273 3415-3426, 2006.

18. Sato K and Kawashima S: Calpain function in the modulation of signal transduction molecules. Biol Chem 382: 743-751, 2001.

19. Hong JM, Teitelbaum SL, Kim TH, Ross FP, Kim SY and Kim HJ: Calpain-6, a target molecule of glucocorticoids, regulates osteoclastic bone resorption via cytoskeletal organization and microtubule acetylation. J Bone Miner Res 26: 657-665, 2011.

20. Tonami K, Kurihara Y, Arima S, Nishiyama K, Uchijima Y, Asano T, Sorimachi $\mathrm{H}$ and Kurihara $\mathrm{H}$ : Calpain-6, a microtubule-stabilizing protein, regulates Rac1 activity and cell motility through interaction with GEF-H1. J Cell Sci 124: 1214-1223, 2011.

21. Tonami K, Hata S, Ojima K, Ono Y, Kurihara Y, Amano T, Sato T, Kawamura Y, Kurihara H and Sorimachi H: Calpain-6 deficiency promotes skeletal muscle development and regeneration. PLoS Genet 9: e1003668, 2013.

22. Margiotta A, Progida C, Bakke O and Bucci C: Rab7a regulates cell migration through Rac1 and vimentin. Biochim Biophys Acta Mol Cell Res 1864: 367-381, 2017.

23. Rodriguez OC, Schaefer AW, Mandato CA, Forscher P, Bement WM and Waterman-Storer CM: Conserved microtubule-actin interactions in cell movement and morphogenesis. Nat Cell Biol 5: 599-609, 2003.
24. Mogessie B, Roth D, Rahil Z and Straube A: A novel isoform of MAP4 organises the paraxial microtubule array required for muscle cell differentiation. Elife 4: e05697, 2015.

25. Bryan BA, Li D, Wu X and Liu M: The Rho family of small GTPases: Crucial regulators of skeletal myogenesis. Cell Mol Life Sci 62: 1547-1555, 2005

26. Skubitz KM and Skubitz AP: Differential gene expression in uterine leiomyoma. J Lab Clin Med 141: 297-308, 2003

27. Marion A, Dieudonné FX, Patiño-Garcia A, Lecanda F, Marie PJ and Modrowski D: Calpain- 6 is an endothelin-1 signaling dependent protective factor in chemoresistant osteosarcoma. Int J Cancer 130: 2514-2525, 2012.

28. Liu Y, Wang Y, Sun X, Mei C, Wang L, Li Z and Zha X: miR-449a promotes liver cancer cell apoptosis by downregulation of Calpain 6 and POU2F1. Oncotarget 7: 13491-13501, 2016

29. Su X, Xiao D, Huang L, Li S, Ying J, Tong Y, Ye Q, Mu D and Qu Y: MicroRNA alteration in developing rat oligodendrocyte precursor cells induced by hypoxia-ischemia. J Neuropathol Exp Neurol 78: 900-909, 2019.

30. Mahaman YAR, Huang F, Kessete Afewerky H, Maibouge TMS, Ghose B and Wang X: Involvement of calpain in the neuropathogenesis of Alzheimer's disease. Med Res Rev 39: 608-630, 2019.

31. Peng P, Wu W, Zhao J, Song S, Wang X, Jia D, Shao M, Zhang M, Li L, Wang L, et al: Decreased expression of Calpain-9 predicts unfavorable prognosis in patients with gastric cancer. Sci Rep 6: 29604, 2016.

32. Fichna JP, Macias A, Piechota M, Korostyński M, PotulskaChromik A, Redowicz MJ and Zekanowski C: Whole-exome sequencing identifies novel pathogenic mutations and putative phenotype-influencing variants in Polish limb-girdle muscular dystrophy patients. Hum Genomics 12: 34, 2018.

33. Stumvoll M, Goldstein BJ and van Haeften TW: Type 2 diabetes: Principles of pathogenesis and therapy. Lancet 365: 1333-1346, 2005.

34. Miyazaki T, Taketomi Y, Takimoto M, Lei XF, Arita S Kim-Kaneyama JR, Arata S, Ohata H, Ota H, Murakami M and Miyazaki A: m-Calpain induction in vascular endothelial cells on human and mouse atheromas and its roles in VE-cadherin disorganization and atherosclerosis. Circulation 124: 2522-2532, 2011.

35. Matsushita Y, Shimada Y, Kawara S, Takehara K and Sato S Autoantibodies directed against the protease inhibitor calpastatin in psoriasis. Clin Exp Immunol 139: 355-362, 2005.

36. Ono Y, Saido TC and Sorimachi H: Calpain research for drug discovery: Challenges and potential. Nat Rev Drug Discov 15: 854-876, 2016

37. Drag $M$ and Salvesen GS: Emerging principles in protease-based drug discovery. Nat Rev Drug Discov 9: 690-701, 2010.

38. Nikkel AL, Martino B, Markosyan S, Brederson JD, Medeiros R, Moeller A and Bitner RS: The novel calpain inhibitor A-705253 prevents stress-induced tau hyperphosphorylation in vitro and in vivo. Neuropharmacology 63: 606-612, 2012.

39. Ono Y, Ojima K, Shinkai-Ouchi F, Hata S and Sorimachi H: An eccentric calpain, CAPN3/p94/calpain-3. Biochimie 122: 169-187, 2016.

40. Skubitz KM and Skubitz AP: Differential gene expression in leiomyosarcoma. Cancer 98: 1029-1038, 2003.

41. Marion A, Dieudonné F, Patiño-García A, Lecanda F, Marie P and Modrowski D: Identification of calpain- 6 as a new target involved in cell death of bone cancer cells. Bone 44: S247, 2009.

42. Xiang Y, Li F, Wang L, Zheng A, Zuo J, Li M, Wang Y, Xu Y, Chen $\mathrm{C}$, Chen S, et al: Decreased calpain 6 expression is associated with tumorigenesis and poor prognosis in HNSCC. Oncol Lett 13: 2237-2243, 2017.

43. Filali H, Vidal E, Bolea R, Márquez M, Marco P, Vargas A, Pumarola M, Martin-Burriel I and Badiola JJ: Gene and protein patterns of potential prion-related markers in the central nervous system of clinical and preclinical infected sheep. Vet Res 44: 14, 2013.

44. Markmann A, Schäfer S, Linz W, Löhn M, Busch AE and Wohlfart P: Down-regulation of calpain 9 is linked to hypertensive heart and kidney disease. Cell Physiol Biochem 15: 109-116, 2005.

45. Guttula SV, Rao AA, Sridhar GR, Chakravarthy MS, Nageshwararo K and Rao PV: Cluster analysis and phylogenetic relationship in biomarker identification of type 2 diabetes and nephropathy. Int J Diabetes Dev Ctries 30: 52-56, 2010.

46. Miyazaki T, Tonami K, Hata S, Aiuchi T, Ohnishi K, Lei XF, Kim-Kaneyama JR, Takeya M, Itabe $\mathrm{H}$, Sorimachi $\mathrm{H}$, et al: Calpain- 6 confers atherogenicity to macrophages by dysregulating pre-mRNA splicing. J Clin Invest 126: 3417-3432, 2016. 
47. Yin SJ, Lee JR, Kwak H, Lee BN, Han JW, Hahn MJ, Park YD and Yang JM: Functional study of 14-3-3 protein epsilon (YWHAE) in keratinocytes: Microarray integrating bioinformatics approaches. J Biomol Struct Dyn 38: 2633-2649, 2020.

48. Xia L, Liu Y, Fu Y, Dongye S and Wang D: Integrated analysis reveals candidate mRNA and their potential roles in uterine leiomyomas. J Obstet Gynaecol Res 43: 149-156, 2017.

49. Zhu L, Sun Y, Wu Q, Zhang C and Ling J: CAPN6 regulates uterine leiomyoma cell proliferation and apoptosis through the Rac1-dependent signaling pathway. Ann Clin Lab Sci 50: 24-30, 2020.

50. Lee SJ, Choi YL, Lee EJ, Kim BG, Bae DS, Ahn GH and Lee JH: Increased expression of calpain 6 in uterine sarcomas and carcinosarcomas: An immunohistochemical analysis. Int J Gynecol Cancer 17: 248-253, 2007.

51. Rho SB, Byun HJ, Park SY and Chun T: Calpain 6 supports tumorigenesis by inhibiting apoptosis and facilitating angiogenesis. Cancer Lett 271: 306-313, 2008.

52. Lee SJ, Kim BG, Choi YL and Lee JW: Increased expression of calpain 6 during the progression of uterine cervical neoplasia: Immunohistochemical analysis. Oncol Rep 19: 859-863, 2008.

53. LoRusso PM: Inhibition of the PI3K/AKT/mTOR pathway in solid tumors. J Clin Oncol 34: 3803-3815, 2016.

54. Wu XX and Kakehi Y: Enhancement of lexatumumab-induced apoptosis in human solid cancer cells by Cisplatin in caspase-dependent manner. Clin Cancer Res 15: 2039-2047, 2009.

55. Grgic I, Eichler I, Heinau P, Si H, Brakemeier S, Hoyer J and Köhler R: Selective blockade of the intermediate-conductance $\mathrm{Ca}^{2+}$-activated $\mathrm{K}^{+}$channel suppresses proliferation of microvascular and macrovascular endothelial cells and angiogenesis in vivo. Arterioscler Thromb Vasc Biol 25: 704-709, 2005.

56. Tomatis C, Fiorio Pla A and Munaron L: Cytosolic calcium microdomains by arachidonic acid and nitric oxide in endothelial cells. Cell Calcium 41: 261-269, 2007.

57. Apte RS, Chen DS and Ferrara N: VEGF in signaling and disease: Beyond discovery and development. Cell 176: 1248-1264, 2019.

58. Li J, Meng X, Hu J, Zhang Y, Dang Y, Wei L and Shi M: Heparanase promotes radiation resistance of cervical cancer by upregulating hypoxia inducible factor 1 . Am J Cancer Res 7 : 234-244, 2017.

59. Oh M, Rho SB, Son C, Park K and Song SY: Non-proteolytic calpain-6 interacts with VEGFA and promotes angiogenesis by increasing VEGF secretion. Sci Rep 9: 15771, 2019.

60. Marion A, Dieudonné FX, Marie PJ and Modrowski D Endothelin-1 up regulates the survival factor calpain-6 in osteosarcoma cells through Mapk and PI3K pathways. Bone 47: S112, 2010.

61. Marion A, Dieudonné FX, Patiño-Garcia A, Lecanda F, Marie PJ and Modrowski D: Abnormal expression of calpain-6 due to endothelin-1/NFkB signalling contributes to cell survival and chemoresistance in osteosarcoma cells. Bone 48: S38, 2011.

62. Jin W, Wu L, Liang K, Liu B, Lu Y and Fan Z: Roles of the PI-3K and MEK pathways in Ras-mediated chemoresistance in breast cancer cells. Br J Cancer 89: 185-191, 2003.

63. Geismann C, Schäfer H, Gundlach JP, Hauser C, Egberts JH, Schneider G and Arlt A: NF- $\kappa$ B dependent chemokine signaling in pancreatic cancer. Cancers (Basel) 11: 1445, 2019.

64. Andrique C, Morardet L, Linares LK, Cissé MY, Merle C, Chibon F, Provot S, Haÿ E, Ea HK, Cohen-Solal M and Modrowski D: Calpain-6 controls the fate of sarcoma stem cells by promoting autophagy and preventing senescence. JCI insight 3: e121225, 2018.

65. Orosco A, Fromigué O, Bazille C, Entz-Werle N, Levillain P, Marie PJ and Modrowski D: Syndecan-2 affects the basal and chemotherapy-induced apoptosis in osteosarcoma. Cancer Res 67: 3708-3715, 2007.

66. Dieudonné FX, Marion A, Marie PJ and Modrowski D: Targeted inhibition of T-cell factor activity promotes syndecan-2 expression and sensitization to doxorubicin in osteosarcoma cells and bone tumors in mice. J Bone Miner Res 27: 2118-2129, 2012.

67. Zhao Y, Liao Q, Zhu Y and Long H: Endothelin-1 promotes osteosarcoma cell invasion and survival against cisplatin-induced apoptosis. Clin Orthop Relat Res 469: 3190-3199, 2011.
68. Kaci I, Mansouri R, Marie P and Modrowski D: Hypoxia upregulates calpain-6 expression in osteosarcoma cells: Implication in cancer stem cells. J Bone Min Res: 28, 2013.

69. Bouanga JT, Yoon J and Modrowski D: Contribution of cancer stem cells to the metastatic capacities of osteosarcoma. Calcif Tissue Int 104: S85, 2019.

70. Zha X: The PI3K-Akt pathway regulates calpain 6 expression, proliferation, and apoptosis. FASEB J: 25, 2011.

71. Singh NK: microRNAs databases: Developmental methodologies, structural and functional annotations. Interdiscip Sci 9: 357-377, 2017.

72. Wong CM, Tsang FH and Ng IO: Non-coding RNAs in hepatocellular carcinoma: Molecular functions and pathological implications. Nat Rev Gastroenterol Hepatol 15: 137-151, 2018.

73. Raimbourg Q, Perez J, Vandermeersch S, Prignon A, Hanouna G, Haymann JP, Baud L and Letavernier E: The calpain/calpastatin system has opposing roles in growth and metastatic dissemination of melanoma. PLoS One 8: e60469, 2013.

74. Birch D, Britt BC, Dukes SC, Kessler JA and Dizon ML: MicroRNAs participate in the murine oligodendroglial response to perinatal hypoxia-ischemia. Pediatr Res 76: 334-340, 2014.

75. Guo Y, Gong HS, Zhang J, Xie WL, Tian C, Chen C, Shi Q, Wang SB, Xu Y, Zhang BY and Dong XP: Remarkable reduction of MAP2 in the brains of scrapie-infected rodents and human prion disease possibly correlated with the increase of calpain. PLoS One 7: e30163, 2012.

76. Yadavalli R, Guttmann RP, Seward T, Centers AP, Williamson RA and Telling GC: Calpain-dependent endoproteolytic cleavage of PrPSc modulates scrapie prion propagation. J Biol Chem 279: 21948-21956, 2004.

77. Donkor I: An update on the therapeutic potential of calpain inhibitors: A patent review. Expert Opin Ther Pat: 1-17, 2020

78. Miyazaki T and Miyazaki A: Dysregulation of calpain proteolytic systems underlies degenerative vascular disorders. J Atheroscler Thromb 25: 1-15, 2018.

79. Miyazaki T and Miyazaki A: Emerging roles of calpain proteolytic systems in macrophage cholesterol handling. Cell Mol Life Sci 74: 3011-3021, 2017.

80. Miyazaki T and Miyazaki A: Impact of dysfunctional protein catabolism on macrophage cholesterol handling. Curr Med Chem 26: 1631-1643, 2019.

81. Kang MY, Zhang Y, Matkovich SJ, Diwan A, Chishti AH and Dorn GW II: Receptor-independent cardiac protein kinase Calpha activation by calpain-mediated truncation of regulatory domains. Circ Res 107: 903-912, 2010.

82. Taneike M, Mizote I, Morita T, Watanabe T, Hikoso S, Yamaguchi O, Takeda T, Oka T, Tamai T, Oyabu J, et al: Calpain protects the heart from hemodynamic stress. J Biol Chem 286: 32170-32177, 2011

83. Galvez AS, Diwan A, Odley AM, Hahn HS, Osinska H, Melendez JG, Robbins J, Lynch RA, Marreez Y and Dorn GW II: Cardiomyocyte degeneration with calpain deficiency reveals a critical role in protein homeostasis. Circ Res 100: 1071-1078, 2007.

84. Moyen C, Goudenege S, Poussard S, Sassi A, Brustis J and Cottin P: Involvement of micro-calpain (CAPN 1) in muscle cell differentiation. Int J Biochem Cell Biol 36: 728-743, 2004.

85. Sáenz A, Azpitarte M, Armañanzas R, Leturcq F, Alzualde A, Inza I, García-Bragado F, De la Herran G, Corcuera J, Cabello A, et al: Gene expression profiling in limb-girdle muscular dystrophy 2A. PLoS One 3: e3750, 2008.

86. Hollinger K and Selsby J: The physiological response of protease inhibition in dystrophic muscle. Acta Physiol (Oxf) 208: 234-244, 2013.

87. Raaby L, Otkjær K, Salvskov-Iversen ML, Johansen C and Iversen L: Characterization of the expression of 14-3-3 isoforms in psoriasis, basal cell carcinoma, atopic dermatitis and contact dermatitis. Dermatol Rep 2: e14, 2010.

This work is licensed under a Creative Commons Attribution-NonCommercial-NoDerivatives 4.0 International (CC BY-NC-ND 4.0) License. 